\title{
Safety and Yield of Early Cessation of AEDs in Video-EEG Telemetry and Outcomes
}

\author{
Farzad Moien-Afshari, Robert Griebel, Venkat Sadanand, Mirna Vrbancic, \\ Lizbeth Hernandez-Ronquillo, Noel Lowry, José F. Téllez Zenteno
}

\begin{abstract}
Background: Video-electroencephalography (VEEG) telemetry is the simultaneous recording of ictal and interictal EEG pattern and paroxysmal behavior to investigate the nature of paroxysmal events. Methods: This is a prospective study performed to asses the safety and yield of early discontinuation of antiepileptic drugs (AEDs) in the telemetry unit. Over a 2.5-year period, 50 patients that met the indications for VEEG monitoring were admitted by an epileptologist to neuro-observation units with continuous monitoring, nursing coverage and EEG technicians support during working hours and on-call thereafter. In most cases AEDs (except Phenobarbital) were discontinued in $24 \mathrm{~h}$. We prospectively assessed the yield and safety of the telemetry investigation as well as epilepsy surgery outcomes. Results: Our monitoring answered the study question in $88 \%$ of the patients. The question was not answered in $12 \%$ of cases due to the lack of recorded events. Our results changed the management in $74 \%$ of cases and potentially improved quality of life by decreasing the AEDs consumption and number of seizures per month. Over all, 22\% received epilepsy surgery and became either seizure free or their seizures became non-disabling. Our method significantly decreased the duration of hospital admission for monitoring and minimal complications occurred only in $8 \%$ of patients. Conclusions: In conclusion, our method for short VEEG monitoring has a high yield for diagnosis, minimal complications and is cost effective. These qualities, together with good surgery results validate our method for the investigation and treatment of refractory seizure cases.
\end{abstract}

RÉSUMÉ: Sécurité, rendement et résultats de l'arrêt précoce des médicaments antiépileptiques quand la télémétrie vidéo EEG est utilisée. Contexte : La vidéoélectroencéphalographie (VEEG) par télémétrie est l'enregistrement simultané de tracés EEG pendant et entre les crises et l'enregistrement du comportement paroxystique, principalement dans le but d'étudier la nature des événements paroxystiques. Méthodes : Il s'agit d'une étude prospective effectuée dans le but d'évaluer la sécurité et le bénéfice d'un arrêt précoce des médicaments antiépileptiques (MAE) à l'unité de télémétrie. Cinquante patients qui satisfaisaient aux indications pour la surveillance par VEEG ont été admis par un épileptologue à une unité d'observation neurologique avec surveillance continue, soins infirmiers et soutien technique EEG pendant les heures de travail et en astreinte par la suite. Dans la plupart des cas, les MAE (excepté le phénobarbital) étaient cessés au cours d'une période de 24 heures. Nous avons évalué de façon prospective le rendement et la sécurité de l'étude par télémétrie ainsi que les résultats de la chirurgie pour épilepsie. Résultats : Notre surveillance a fourni la réponse chez $88 \%$ des patients alors que chez $12 \%$ des patients l'information n'a pas été obtenue parce qu'aucun événement n'a été enregistré. Nos résultats ont modifié la décision thérapeutique chez $74 \%$ des patients et ont possiblement amélioré la qualité de vie en diminuant la prise de MAE et le nombre de crises par mois. Dans l'ensemble, $22 \%$ ont subi une chirurgie pour épilepsie et n'ont plus présenté de crises ou leurs crises n'étaient plus invalidantes. Notre méthode a diminué significativement la durée d'hospitalisation pour surveillance et seulement $8 \%$ des patients ont présenté des complications mineures. Conclusions : Notre méthode de surveillance VEEG brève a un rendement diagnostique élevé, des complications minimes et elle est efficiente. Ces qualités ainsi que de bons résultats chirurgicaux valident notre méthode d'évaluation et de traitement des cas d'épilepsie réfractaire.

Can. J. Neurol. Sci. 2009; 36: 587-592

Video-electroencephalography (VEEG) telemetry is the simultaneous recording of ictal and interictal EEG pattern and paroxysmal behavior. This technique is used in adults for different purposes including: identification of the nature of paroxysmal events, verification of the diagnosis of seizure disorder, categorization seizure type(s), evaluation of frequency of seizure or interictal epileptiform discharges, and probably the most significant use is identification of candidates for epilepsy surgery (patients having poor response to medical therapy) by localizing seizures ${ }^{1-7}$.

A reliable recording system and a team of devoted, skillful nursing (operating 24 hours) and technical staff (on-call after working hours) are required for a successful unit. Antiepileptic

\footnotetext{
From the Department of Medicine-Division of Neurology (FMA, LHR, JFTZ), Department of Neurosurgery (RG, VS), Department of Pathology and Laboratory Medicine (MV), Department of Pediatrics (NL), University of Saskatchewan, Saskatoon, SK, Canada.

Received January 28, 2009. Final Revisions Submitted April 29, 2009. Correspondence to: José F. Téllez Zenteno, Department of Medicine, Division of Neurology, Royal University Hospital, Saskatoon, Saskatchewan, S7N 0W8, Canada. E-mail: jft084@mail.usask.ca
} 
drug (AED) tapering with or without sleep deprivation may be used to precipitate seizures. Especially in potential candidates for epilepsy surgery, AED withdrawal is useful since it affects seizure propagation instead of seizure pacemaker ${ }^{8}$. This increases the risk of provoked secondary generalized seizures with an early peak that for some medications could be a rebound phenomenon in addition to loss of therapeutic effect ${ }^{9}$. Generalized tonic clonic seizures in acute withdrawal may occasionally end in refractory status epilepticus ${ }^{10}$. Therefore, continuous supervision of patients, and the ability to respond rapidly to a seizure are essential for patient safety ${ }^{1}$. Despite the existence of the guidelines of the National Association of Epilepsy Centers for standards of care in specialized epilepsy centers $^{11}$, several safety issues have been raised in units across the United States of America. Therefore, a panel of experts recently studied the safety in VEEG telemetry units. ${ }^{12}$ The preliminary results indicate some of the possible causes of variable safety in different VEEG telemetry units including lowered AEDs dosage prior to admission, lack of continuous monitoring by the nursing or EEG staff, and absence of a written protocol for the treatment of status epilepticus or speed of medication withdrawal after admission ${ }^{12}$. The requirement of special resources and personnel, which was further emphasized by the current report on the safety of the units ${ }^{12}$, and the prolonged hospitalization that is inherent to VEEG telemetry recordings lead to a high cost which is one of the disadvantages of this technique ${ }^{2}$. Therefore, finding strategies that can safely optimize this technique to decrease the cost are extremely important.

Telemetry investigations at the Royal University Hospital in Saskatoon are performed in a safely monitored unit with a system of rapid tapering of patients' AEDs plus early sleep deprivation in most patients to provoke seizures in a shorter admission time. The application of this method in a safe environment and on a routine basis will significantly lower the cost for VEEG monitoring. However, the safety and diagnostic yield of this system needs to be further established which are the main objectives of this study. We also looked at the outcome of epilepsy surgeries since a high number of seizure free patients post-surgery is indirectly related to the efficacy of our monitoring system in determination of surgery candidacy and seizure localization despite the short recording. Another aim of the study was to assess if the patients with medically intractable seizures were referred to the epileptologist in a timely manner.

\section{Methods}

Over a two and a half year period (2006-2008), patients who met the indications for VEEG monitoring were admitted by an adult epileptologist to Royal University Hospital, Saskatoon, Saskatchewan and were monitored in the neurology ward observation units. Each observation unit was a room without partitions, located on the neurology floor, and included four hospital beds around a nursing station. Two registered nurses with specialized neurological training were physically present in the unit 24 hours a day, seven days a week and could directly observe the patient and the EEG monitor at the bed side. Only one of the four beds at each observation unit was occupied by a VEEG telemetry patient at a time and the other beds were occupied by patients with different neurological diagnoses. The unit nurses monitored patients' vital signs, including neurovital signs, every two hours. Electroencephalography technicians and the epileptologist were available at the hospital five days a week during working hours and on-call thereafter. Video-EEG recording was continuous throughout the admission and nurses were able to page the on-call EEG technician (for technical support) and the on-call neurology resident and/ or the epileptologist (for medical support) after hours. In patients without past history of status epilepticus, antiepileptic drugs (AEDs) (except Phenobarbital) were decreased to half of the dose on admission and fully discontinued at 24 hours (early discontinuation). If history of status epilepticus was positive, AEDs (except Phenobarbital) were tapered daily by $25 \%$ of the initial dose to none. Antiepileptic drugs were never tapered prior to admission. To prevent status epilepticus, a written protocol for administration of intravenous lorazepam (2 $\mathrm{mg}$ within five minutes of seizure onset and repeat every five minutes for two doses as needed) was placed for all patients on admission. In two

Table 1: Summary of reasons for admission and conclusions of VEEG telemetry in 50 patients

\begin{tabular}{llll}
\hline Reason for admission & \# of patients (\%) & Conclusion & \# of patients (\%) \\
\hline \multirow{2}{*}{ Presurgical } & $20(40 \%)$ & $\begin{array}{l}\text { Surgery without intracranial recording } \\
\text { (IR) }\end{array}$ & $15(30 \%)$ \\
Diagnostic & $30(60 \%)$ & PNES only & $12(24 \%)$ \\
& & Intracranial recording needed & $8(16 \%)$ \\
& Inconclusive & $6(12 \%)$ \\
& & PNES and epilepsy & $3(6 \%)$ \\
& & PGE & $5(10 \%)$ \\
& & Sleep Disorder & $1(2 \%)$ \\
\hline Total & & Total & $50(100 \%)$ \\
\hline
\end{tabular}

The specific reasons of admission are displayed in the text. PNES: psychogenic non-epileptic spells, PGE: primary generalized epilepsy. 


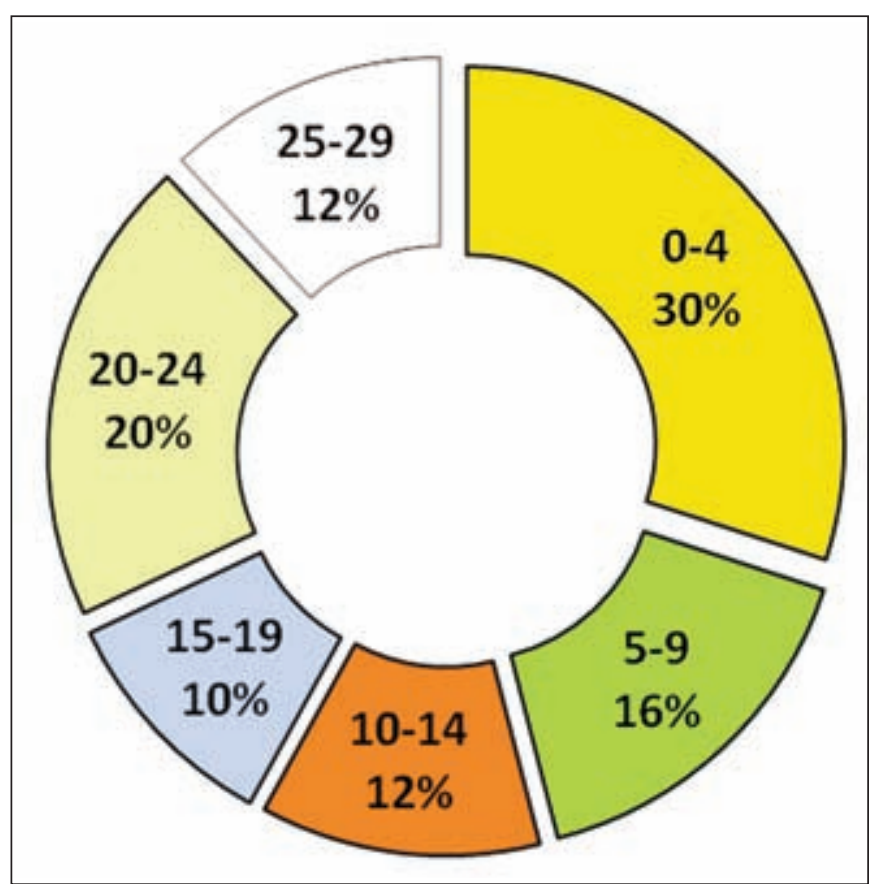

Figure 1: Number of years with spells prior to referral to our epilepsy center.

patients intracranial subdural recording was performed and in both cases AEDs were discontinued in 24 hours. Overnight sleep deprivation was supervised by the nursing staff. To avoid sleep, patients alternatively watched movies or played video, board or card games with a family member. Three seizures were the minimum requirement to conclude the study and the classification by International League Against Epilepsy was used to classify epilepsy syndromes and seizures. Non-epileptic spells were diagnosed when the clinical events were not accompanied by ictal EEG changes. Patients with focal onset epilepsy were referred for epilepsy surgery. The main outcomes of our study were assessed prospectively and patients were followed for a minimum of one year post VEEG telemetry and/ or epilepsy surgery. The complications that were considered significant for the assessment of safety were status epilepticus, traumatic falls, cluster of seizures within one month after discharge, and major injuries such as fractures. The yield of the VEEG telemetry investigation was assessed as the percentage of the prerecording questions that were answered upon completion of the investigation. Our protocol was approved by the ethics committee of the Royal University Hospital.

Descriptive statistics were used to describe the basic features of the data gathered from our study. Statistical analysis was performed with SPSS v16 for Windows. T-test and "proportion comparison test" were used for comparison of results with previous reports where appropriate. Microsoft excel 2007 was used for graph construction.

\section{Results}

In total, 50 patients (52\% males) were monitored in the determined period. Patients' age was between 17 and 80 years (mean: 36.3, SD: 14.3) and the majority (70\%) were younger than 30 years. Onset of seizure was at 20 years or younger in $60 \%$ (mean: 22.5, SD: 19.3). More than half of the patients $(54 \%)$ had intractable spells for more than nine years before referral to our center (Figure 1). Neurological exam was normal in $90 \%$ and routine EEG was normal in $24 \%$ of patients prior to admission. Table 1 contains VEEG telemetry admission indications versus final conclusions based on our findings.

The mean number of spells reported by patients before admission per month were 12 (1 - 100) (Figure 2A), average years with seizure were $12.2(0.1-27)$. Thirty one patients had at least one prior emergency admission and ten had previous intensive care unit admissions. The average number of AEDs used was 1.86 (SD: 1.12, 0 - 4) (Figure 2B). The most common brain MRI diagnosis was normal $(52 \%)$, and MRI was not performed in $10 \%$ of patients.

Conservative tapering (25\% per day) of AEDs was used only

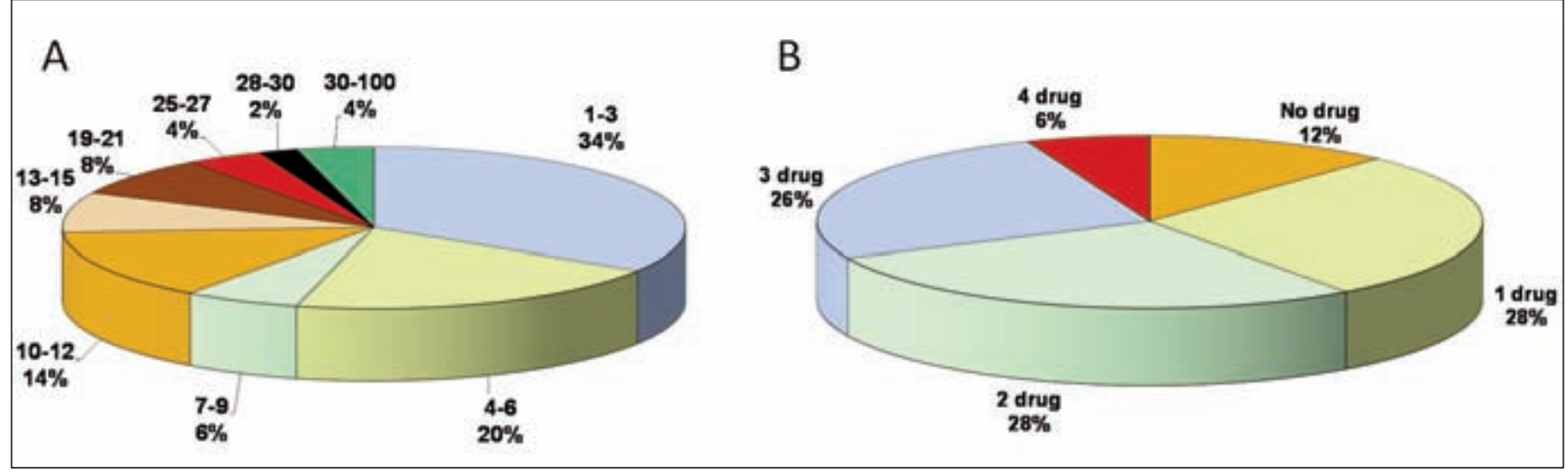

Figure 2: A-Number of spells reported by patients per month prior to admission. B-Number of AEDs used before admission. 
Table 2: Type of AEDs, average dosage, dosage range, number and percentage of patients receiving the medications

\begin{tabular}{|c|c|c|c|}
\hline Medication & Mean Dose \pm SD & Dosage range (mg od) & $\begin{array}{c}\# \text { of } \\
\text { patients } \\
(\%)\end{array}$ \\
\hline Phenytoin & $343 \pm 87$ & $250-600$ & $14(28)$ \\
\hline Carbamazepine & $1213 \pm 410$ & $400-2000$ & $15(30)$ \\
\hline Levetiracetam & $2084 \pm 790$ & $1000-3250$ & $16(32)$ \\
\hline Topiramate & $275 \pm 123$ & $100-500$ & $10(20)$ \\
\hline Lamotrigine & $329 \pm 166$ & $100-600$ & $12(24)$ \\
\hline Valproic acid & $1500 \pm 0$ & 1500 & $4(8)$ \\
\hline Phenobarbital & $206 \pm 108$ & $105-300$ & $4(8)$ \\
\hline Clobazam & $21 \pm 9$ & $10-40$ & $13(26)$ \\
\hline Gabapentin & $900 \pm 520$ & $300-1200$ & $3(6)$ \\
\hline Pregabalin & $225 \pm 106$ & $150-300$ & $2(4)$ \\
\hline
\end{tabular}

in eight patients due to prior history of status epilepticus or if they were on Phenobarbital (not discontinued), one patient was not on AEDs, and in the remaining 41 patients AEDs were discontinued in 24 hours as per methods. Patients' preadmission AED types and dosages are summarized in Table 2. Over night sleep deprivation was applied on the second or third day of telemetry in 38 patients.

On average, five seizures ( 1 - 50) and 3.5 non-epileptic events (1 - 65) were recorded per patient. Mean hospital stay for monitoring was 4.4 days (SD: $2,1-10)$ and in 44 patients (88\%) the monitoring was conclusive in five days or less. Of the 44 patients in whom spells were recorded (including seizures and non epileptic events), $90 \%$ had spells between the first and the second day. On the third day, all the patients had a spell or spells.

In 31 patients, epileptic events were recorded (Figure 3, dark grey ovals). Of these 31 patients, 23 were identified as candidates for epilepsy surgery. Of these 23, eight needed intracranial recording and 15 patients were identified as definite candidates for epilepsy surgery without the necessity to perform intracranial investigation (i.e. intractable focal epileptiform activity was recorded). In the latter group, epilepsy surgery was done on 11 patients (Figure 3), and it was not performed in four patients, but they are on the waiting list for surgery. Of the 11 patients who received surgery, 8 became seizure free or Engel class I, and 2 have rare disabling seizures or Engel class II after one year follow-up. In the remaining 8 of these 31 patients, the diagnosis was elucidated; however, these were not surgical candidates (Figure 3). These included: Non-epileptic evens plus epilepsy (3), primary generalized epilepsy (5), confirmation of epilepsy diagnosis (2), we were able to answer the question in $88 \%$ of patients.

In 16 patients no epileptic events were recorded (Figure 3, light gray and medium gray ovals). Of these 16,13 patients had

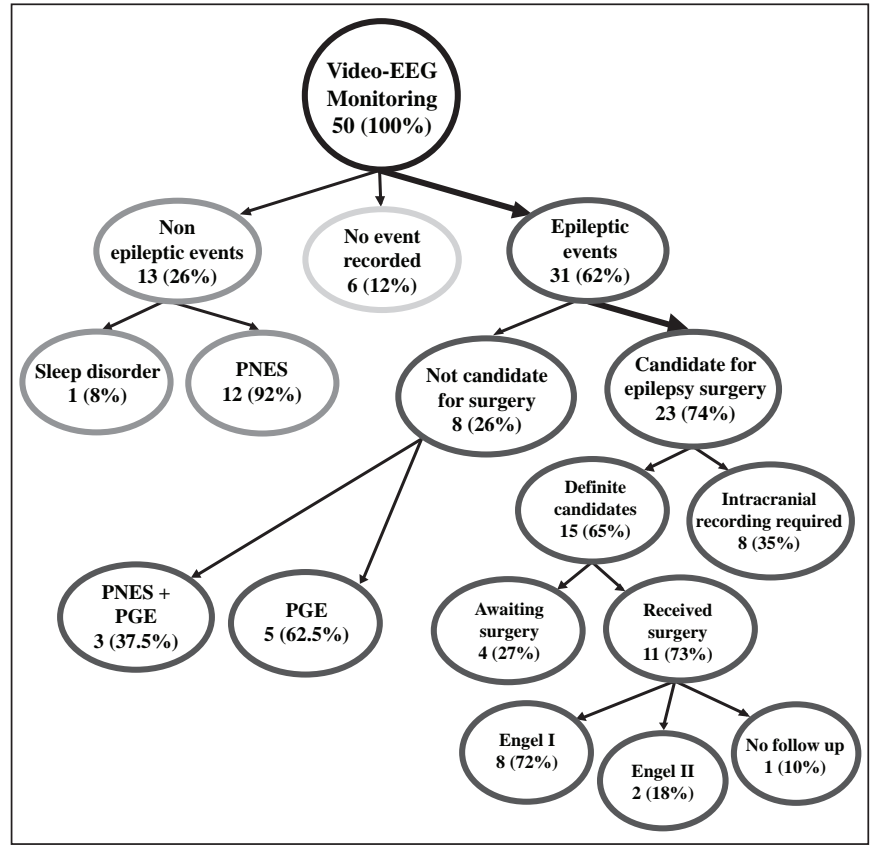

Figure 3: Distribution of video-EEG monitoring diagnoses and outcome (PNES: psychogenic nonepileptic seizures, PGE: primary generalized epilepsy). Majority of monitored patients had epileptic events and most of these became candidates for epilepsy surgery (thicker arrows). Engel Class I (free of disabling seizures) and Class II (rare disabling seizures)

non-epileptic events of which 12 had psychogenic non-epileptic seizures (PNESs), and one had a sleep disorder. In 6 patients no event (including non-epileptic) was recorded (Figure 3, light 
grey oval). Patients with PNESs were referred to mental health professionals and their antiepileptic drug was discontinued.

Complications of telemetry only occurred in four patients, which included non-traumatic fall, back pain, skin infection at the site of intravenous catheter and admission to emergency within one month of telemetry due to a cluster of seizures which were controlled without admission to intensive care unit. The patient with the skin infection was treated with antibiotics without complications. The patient with the back pain had Xrays and MRI and no major abnormality was found.

\section{Discussion}

The main objectives of this study were to assess the safety and diagnostic yield of rapid tapering of patients' antiepileptic medication(s) plus early sleep deprivation to provoke seizures in a shorter VEEG telemetry admission period. We also looked at the outcome of epilepsy surgeries and the delay between seizure onset and referral to the epilepsy center. Overall, we monitored 50 patients for an average of 4.4 days and answered the study question in 44 of them. Our results changed the management in $74 \%$ of the patients towards improving their quality of life. Minor complications occurred only in four patients.

Our findings indicate that the method used in this study is relatively safe. Our complication rate was only $8 \%$, which is comparable to what was previously reported $(10.1 \%)^{13}$. The complications in our patients were mainly minor (non-traumatic fall, back sprain, intravenous site infection, cluster of seizures within one week of discharge requiring admission to emergency) compared to those reported by others (status epilepticus, injuries, infections and hemorrhages, cardiac events and post-ictal psychosis $)^{13,14}$. Some possible reasons for the lower rate and severity of complications in our unit are continuous and inperson monitoring of all patients by the highly trained nursing staff, written protocol for administration of antiseizure medication within five minutes of seizure onset to prevent status epilepticus, and finally no tapering of AEDs prior to admission. Our short stay protocol was initially adapted due to inavailability of our EEG technicians over the weekends; however, the method turned out to be safe and efficient.

The result of our monitoring changed the management and potentially improved quality of life in $74 \%$ of the cases. These were patients that were mostly young with intractable seizures (more than three spells per month) and on polypharmacy (two or more AEDs) and therefore their quality of life was negatively affected due to the potential limitation of their activities. As a result of VEEG telemetry in these patients, $26 \%$ were able to fully discontinue their AEDs (sleep disorder, PNES), and $22 \%$ received epilepsy surgery and became either seizure free or their seizures became non-disabling. Therefore, our results helped to decrease the quantity of AED consumption and/or the number of seizures per month per patient and as a result potentially improved their quality of life. The excellent outcome of epilepsy surgeries suggests that VEEG telemetry with our method accurately localized the seizure onset.

Our VEEG monitoring was effective despite patients' short stay. Our monitoring answered the study question in $88 \%$ of the cases, which is a higher diagnostic rate compared to some previously published report ${ }^{13}$ at $69.8 \%$ ( $\mathrm{p}<0.001$, proportion comparison test). The higher diagnostic yield in our unit could possibly result from a different patient population (only adults) and rapid discontinuation of AEDs combined with sleep deprivation. Moreover, the non-epileptic events were recorded in $26 \%$ of our patients, which is similar to previous reports (about $20 \%)^{2,3,15,16}$. Finally, $30 \%$ of our monitored patients were definitive candidates for epilepsy surgery, which is comparable to the average rate reported by others $(20 \%)^{1}$.

Our results suggest that our method for VEEG monitoring can lower the cost of in-patient recording by shortening the admission time. We used a method of short stay for VEEG telemetry with rapid tapering and discontinuation of AEDs combined with early sleep deprivation in most patients. As a result, length of hospital stay in our facility was shorter $(4.4 \pm 2$ days) as compared to other telemetry units (8.2 \pm 4.3 days $)(\mathrm{p}<0.001, \mathrm{t} \text { test })^{13}$. While rapid tapering of AEDs provokes seizures and may contribute to decreased length of admission for VEEG monitoring, one may argue that sleep deprivation may not contribute to patients' short stay as it did not have a seizure provoking effect in a previous report ${ }^{17}$. However, in the report by Malow et $\mathrm{al}^{17}$ only patients with medically refractory partial epilepsy were admitted for monitoring, which is different than our admission indications and therefore their results may not apply to our study.

We found an average nine-year delay between seizure onset and referral to our epilepsy center in the majority of our patients, which could partly be related to the absence of an adult epileptologist in the district until recent years. However, similar referral delay has been reported by others ${ }^{18,19}$ and may indicate low awareness amongst clinicians of the indications for VEEG telemetry. This is an important observation since late referral will delay appropriate management and may negatively affect recovery, particularly in patients with PNES, and also the outcome of epilepsy surgery ${ }^{20-23}$.

The main limitation of our study is lack of a concurrent control group and therefore we used historical controls by comparing our results with previous studies. Another limitation of the study is our medium sample size which may underestimate the complications. In conclusion, the results indicate that our method for VEEG monitoring has a high yield for diagnosis, minimal complications and is cost effective. These qualities, together with a good outcome of epilepsy surgery validate our method for the investigation and treatment of refractory seizure cases.

\section{ACKNOWLEDGEMENT}

This research was supported by research grant from the University of Saskatchewan.

\section{REFERENCES}

1. Benbadis SR, O'Neill E, Tatum WO, Heriaud L. Outcome of prolonged video-EEG monitoring at a typical referral epilepsy center. Epilepsia. 2004;45(9):1150-3.

2. Binnie CD, Rowan AJ, Overweg J, Meinardi H, Wisman T, Kamp A, et al. Telemetric EEG and video monitoring in epilepsy. Neurology. 1981;31(3):298-303.

3. Douglas R, Nordli JR. Usefulness of video-EEG monitoring. Epilepsia. 2006;47(S1):26-30.

4. Gotman J, Marciani MG. Electroencephalographic spiking activity, drug levels, and seizure occurrence in epileptic patients. Ann Neurol. 1985;17(6):597-603. 
5. Gotman J, Levtova V, Farine B. Graphic representation of the EEG during epileptic seizures. Electroencephalogr Clin Neurophysiol. 1993;87(4):206-14.

6. Sutula TP, Sackellares JC, Miller JQ, Dreifuss FE. Intensive monitoring in refractory epilepsy. Neurology. 1981;31(3):243-7.

7. Cascino GD. Video-EEG monitoring in adults. Epilepsia. 2002; 43(S3):80-93.

8. Zhou D, Wang Y, Hopp P, Kerling F, Kirchner A, Pauli E, et al. Influence on ictal seizure semiology of rapid withdrawal of carbamazepine and valproate in monotherapy. Epilepsia. 2002; 43(4):386-93.

9. Azar NJ, Wang L, Song Y, Abou-Khalil BW. Temporal pattern of oxcarbazepine and phenytoin withdrawal seizures during epilepsy monitoring. Epilepsy Res. 2008;79(1):78-83.

10. Yen DJ, Chen C, Shih YH, Guo YC, Liu LT, Yu HY, et al. Antiepileptic drug withdrawal in patients with temporal lobe epilepsy undergoing presurgical video-EEG monitoring. Epilepsia. 2001;42(2):251-5.

11. Gumnit RJ, Walczak TS. Guidelines for essential services, personnel, and facilities in specialized epilepsy centers in the United States. Epilepsia. 2001;42(6):804-14.

12. Buelow JM, Privitera M, Levisohn P, Barkley GL. A description of current practice in epilepsy monitoring units. Epilepsy Behav. 2009;15(3):308-13.

13. Wijesuriya M, Jette N, Macrodimitris S, Suddes M, Martini J, Sadiq $\mathrm{S}$, et al. Quality and safety indicators for a seizure monitoring unit [abstract]. Epilepsia. 2007;48 Suppl 6:S28.

14. Noe K, Drazkowski J. Safety of long-term video EEG monitoring [Abstract]. Epilepsia. 2009;49 Suppl 7:S54.

15. Asano E, Pawlak C, Shah A, Shah J, Luat AF, Ahn-Ewing J, et al. The diagnostic value of initial video-EEG monitoring in children--review of 1000 cases. Epilepsy Res. 2005;66(1-3): 129-35.
16. McBride AE, Shih TT, Hirsch LJ. Video-EEG monitoring in the elderly: a review of 94 patients. Epilepsia. 2002;43(2):165-9.

17. Malow BA, Passaro E, Milling C, Minecan DN, Levy K. Sleep deprivation does not affect seizure frequency during inpatient video-EEG monitoring. Neurology. 2002;59(9):1371-4.

18. Benbadis SR, Heriaud L, Tatum WO, Vale FL. Epilepsy surgery, delays and referral patterns-are all your epilepsy patients controlled? Seizure. 2003;12(3):167-70.

19. Reuber M, Fernandez G, Bauer J, Helmstaedter C, Elger CE. Diagnostic delay in psychogenic nonepileptic seizures. Neurology. 2002;58(3):493-5.

20. Benbadis SR, Tatum WO, Vale FL. When drugs don't work: an algorithmic approach to medically intractable epilepsy. Neurology. 2000;55(12):1780-4.

21. Carton S, Thompson PJ, Duncan JS. Non-epileptic seizures: patients' understanding and reaction to the diagnosis and impact on outcome. Seizure. 2003;12(5):287-94.

22. Gudmundsson O, Prendergast M, Foreman D, Cowley S. Outcome of pseudoseizures in children and adolescents: a 6-year symptom survival analysis. Dev Med Child Neurol. 2001;43(8):547-51.

23. Selwa LM, Geyer J, Nikakhtar N, Brown MB, Schuh LA, Drury I. Nonepileptic seizure outcome varies by type of spell and duration of illness. Epilepsia. 2000;41(10):1330-4. 